\title{
Transformation of a hybrid journal into a gold open access journal: Example of the European Journal of Mineralogy

\author{
JANNICK INGRIN
}

Univ. Lille, CNRS, INRAE, ENSCL, UMR 8207 - UMET -

Unité Matériaux et Transformations

Presenting Author: jannick.ingrin@univ-lille.fr

The European Journal of Mineralogy (EJM) is owned by four European learning Societies (DMG, SEM, SFMC and SIMP). The journal, published under a commercial publisher, used the open access hybrid model. Early 2018, it was decided to move to full open access. In this talk, we discuss the transformation challenges and its impact. It took us two years to fully complete this transformation. Since 2020, the journal is published as gold open access by a reputable open access publisher offering very moderate APCs. The quality and attractiveness of the journal has improved considerably, namely: wider dissemination, reduction of the processing time for publication, reduction of the cost of production by more than $50 \%$. Notwithstanding, the first nine months of 2020, the number of submissions decreased compared to previous years, but we are now back to the same submission rate as before. It means that the implementation of APCs, which was new for many authors, has only marginally affected the attractiveness of the journal. Nevertheless, in order to help authors adjusting, the current APCs rates are still below the real cost of production. The nominal cost will be reached only in a few years. The learning societies have also launched APCs waiver programs to help authors who have difficulties to pay full rates.

The open access revolution of EJM is work in progress. We still have to transform the past hybrid volumes into open access formats. This will be implemented as soon as APCs generated funds fully cover the running cost of the journal. Once all EJM volumes are in open access, the cost of a paper published in EJM will be fully transparent to authors, institutions and tax payers. It will correspond exactly to the APCs paid. It was never the case in the previous hybrid system. This is a major achievement thanks to gold open access. 